\title{
Anafilaxia a opioides en un paciente oncológico pediátrico: reporte de caso
}

\section{Anaphylaxia to opioids in a pediatric oncological patient, case report}

Adrián Díaz Medina « 1,a

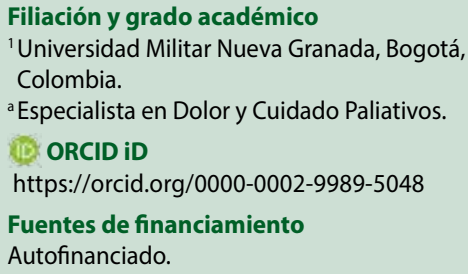

${ }^{a}$ Especialista en Dolor y Cuidado Paliativos.

ORCID iD

https://orcid.org/0000-0002-9989-5048

Fuentes de financiamiento

Autofinanciado.

Conflictos de interés

Declaro que no tengo intereses financieros ni de otro tipo, relacionados con el siguiente artículo.

Recibido: 15-07-2020

Arbitrado por pares

Aceptado: 01-09-2020

Citar como

Díaz A. Anafilaxia a opioides en un paciente oncológico pediátrico: reporte de caso. Rev Peru Cienc Salud. 2020; 2(3): 182-7. doi: http://doi. org/10.37711/rpcs.2020.2.3.197

\section{RESUMEN}

Se presenta el caso de un paciente de 5 años con diagnóstico de leucemia linfoblástica aguda y trisomía 21, quien fue hospitalizado para manejo con quimioterapia de primera línea en quien se presentaron diversas complicaciones durante su tratamiento, por lo que requirió manejo antibiótico y analgésico opioide. Posteriormente, desarrolla cuatro reacciones alérgicas; tres de ellas asociadas a opioides y una catalogada como angioedema severo relacionado con tramadol. Se presenta el caso clínico debido a la baja incidencia de reacciones severas alérgicas a opioides, particularmente al tramadol en el contexto de pacientes de alta complejidad con polimedicación.

Palabras clave: humanos, tramadol, analgésicos, opioides síndrome de Down, incidencia, agentes antibacterianos, trisomía, leucemia-linfoma, linfoblástico de células precursoras, angioedema (Fuente: DeCS - BIREME).

\section{ABSTRACT}

We present a case of a patient with a diagnosis of acute lymphoblastic leukemia and trisomy 21, who was hospitalized for initiation of management with first-line chemotherapy in whom there were various complications during his treatment that required both antibiotic and opioid analgesic management. Subsequently develops four allergic-type reactions, three of them were associated with opioids and the last of them classified as severe angioedema related to the administration of tramadol. The presentation of the clinical case is made, due to the low incidence of occurrence of severe allergic reactions to opioids, particularly to tramadol in the context of a highly complex patient receiving multiple medications.

Keywords: Humans, Tramadol, Analgesics, Opioid, Down Syndrome, Incidence, Anti-Bacterial Agents, Trisomy, Precursor Cell Lymphoblastic, LeukemiaLymphoma, Angioedema (Source: MeSH - NLM). 


\section{INTRODUCCIÓN}

Las alergias son reacciones inmunes exageradas a antígenos, siendo la alergia a opioides una de las más comúnmente reportadas; sin embargo, muchos pacientes han sido clasificados erróneamente como alérgicos ${ }^{(1)}$; Usualmente no son eventos que amenazan la vida como los antibióticos ${ }^{(2)}$, del grupo de las las penicilinas ${ }^{(3)}$. Las reacciones de hipersensibilidad a opioides son raras y se deben a la liberación de histamina no-inmunológica ${ }^{(4)}$. Muchos opioides son potentes liberadores de histamina ${ }^{(5)}$. La codeína y la morfina son más proclives a desencadenar liberación de histamina ${ }^{(6)}$. Hasta el $80 \%$ de los pacientes usuarios de opioides han sido erróneamente catalogados como alérgicos ${ }^{(7)}$.

La anafilaxis se define como la concurrencia de hipotensión y disnea mediada por una respuesta inmune que amenaza la vida (3), causada por liberación súbita de mediadores de los mastocitos en circulación sistémica por inmunoglobulina (lgE) u otros agentes capaces de activar los mastocitos ${ }^{(8)}$. A continuación, presentamos el caso de un paciente en quien se documentaron diversas reacciones de hipersensibilidad; una de ellas de tipo anafiláctico.

\section{CASO CLÍNICO}

Se trata de paciente masculino de 5 años con trisomía del cromosoma 21 y diagnóstico de leucemia linfoblástica aguda de precursores $B$ que ingresó al servicio de oncología pediátrica para aplicación de quimioterapia, con vincristina $1,4 \mathrm{mg} / \mathrm{m}^{2}$, metrotexate $10 \mathrm{mg} / \mathrm{m}^{2}$ y mercaptopurina $0,3 \mathrm{mg} / \mathrm{kg}$.

A los cuatro días de inicio de la terapia se reporta dermatitis de predominio axial, pruriginosa, por lo que se prescribió hidrocortisona $1 \mathrm{mg} / \mathrm{kg}$ y loratadina $0,2 \mathrm{mg} / \mathrm{kg} / \mathrm{dia}$. Hasta ese momento sin uso de opioides o antiinflamatorios no esteroideos (AINE). A los ocho días del ingreso se reporta dolor intenso en cavidad oral por mucositis. Asimismo, se reporta neutropenia febril, por lo cual se indicó antibiótico piperacilina-tazobactam $150 \mathrm{mg} / \mathrm{kg} /$ dia y se inició tramadol a $1 \mathrm{mg} / \mathrm{kg} /$ dosis, que escaló a $3 \mathrm{mg} / \mathrm{kg} /$ dosis en 72 horas, sin mejoría.

Luego de ocho días con esta terapia continua con dolor no controlado, por lo que se reemplazó con morfina $0,05 \mathrm{mg} / \mathrm{kg} /$ dosis para un total de $1 \mathrm{mg}$ cada 6 horas (QID), el 15 de mayo de 2018, sin mejoría; por lo que se aumenta a 2 mg QID.

Al tercer dia de morfina se reporta edema oral, gingival, glótico, labial y extensión maxilar, tos bitonal y eritema. Se decide iniciar el trataamiento de hidrocortisona $1 \mathrm{mg} / \mathrm{kg}$ y cristaloides, se suspende morfina y se cambia a oxicodona 0,1 $\mathrm{mg} / \mathrm{kg} /$ dosis para $2 \mathrm{mg}$ QID.

Al sexto dia de manejo analgésico se cambia a tramadol $2 \mathrm{mg} / \mathrm{kg} /$ dosis para $10 \mathrm{mg}$ IV QID y se retira tres dias después por buen control de síntomas. Luego de una hospitalización de un mes, se completó la quimioterapia y se da salida. Quince días después de la salida, se hopsitaliza para nuevo ciclo de quimioterapia, con vincristina 1,4 $\mathrm{kg} / \mathrm{m} 2$, metrotexate $10 \mathrm{mg} / \mathrm{m}^{2}$ e ifosfamida $1200 \mathrm{mg} /$ $\mathrm{m} 2 /$ dia. El 16 de mayo de 2018 se reporta neutropenia, mucositis y dolor, iniciando tratamiento con paracetamol.

A los nueve dias de iniciar este ciclo, se inicia tratamiento con tramadol $2 \mathrm{mg} / \mathrm{kg}$, por dolor intenso abdominal visceral que se cambió a hidromorfona $0,015 \mathrm{mg} / \mathrm{kg} /$ dosis a los tres días, por mal control de dolor, un día después se diagnostica neutropenia febril y se inicia con carbapenem $25 \mathrm{mg} / \mathrm{kg} /$ dosis y vancomicina $40 \mathrm{mg} / \mathrm{kg} / \mathrm{dia}$. A las 48 horas de iniciada la hidromorfona se reporta edema y eritema facial similar al evento con morfina en la hospitalización previa que se manejó con hidrocortisona $50 \mathrm{mg}$ IV dosis única, se suspendió la hidromorfona y se cambia a tramadol $2 \mathrm{mg} / \mathrm{kg}$. Tres horas después, el paciente presenta deterioro neurológico, falla respiratoria, edema severo en lengua y cuello, considerando edema angioneurótico; ingresando a unidad de cuidados intensivos pediátricos (UCIP). Requiere intubación, ventilación mecánica y cambio a fentanilo $1 \mathrm{mcg} / \mathrm{kg} /$ dosis y dipirona $20 \mathrm{mg} / \mathrm{kg} /$ dosis

Durante el manejo en la UCIP se continuó el esquema antibiótico y se extubó a los seis días. y pasa a sala general de oncología pediátrica. El paciente ha completado 4 ciclos de quimioterapia en manejo solo con acetaminofén $15 \mathrm{mg} / \mathrm{kg} /$ dosis y dipirona $20 \mathrm{mg} / \mathrm{kg} /$ dosis.

\section{DISCUSIÓN}

Al inicio de la quimioterapia, el paciente presentó una reacción cutánea de tipo alérgico sin recibir opioides o AINE, luego de diversas reacciones a opioides. Durante el cuarto evento, el paciente desarrolla angioedema asociado a tramadol, el cual es molecularmente diferente a la morfina, sin embargo, comparten algunas características ${ }^{(9)}$, ya que tiene una estructura fenilpropilamina ${ }^{(5)}$. La incidencia reportada de angioedema por tramadol es de 1 de cada 1000 hasta 1 ede cada 10000 pacientes ${ }^{(9)}$.

Las reacciones alérgicas a tramadol son raras; según Ariosto et al., los eventos alérgicos fueron causados por hidromorfona (34\%), morfina (19\%) y tramadol $(2 \%)^{(2)}$. En un reporte de caso de Mori et al., se presenta 
un paciente con asma alérgica que, luego de recibir tramadol, desarolló anafilaxia e insuficiencia respiratoria, requiriendo de intubación, manejo con corticoesteroide y epinefrina ${ }^{(9)}$.

Hay reportes de reacción tipo I mediada por lgE relacionadas con tramadol ${ }^{(5,28)}$. En Hallberg et al., se hace un reporte de seis casos de angioedema inducido por tramadol ${ }^{(10)}$. El test de provocación de reacciones alérgicas (DPT) es el Gold Standart para el diagnóstico (11,12). Sin embargo, estudios como Nasser et al., reportan que las pruebas en piel no son útiles en la práctica clínica para predecir una alergia a opioides $(13,20,23,24,26)$.

El uso de pruebas cutáneas es cuestionable porque los opioides pueden liberar histamina y los resultados no son diferentes entre los verdaderos alérgicos y los controles. Además, el valor específico de la prueba de IgE ha sido cuestionado porque puede ser positivo en el $10 \%$ de los pacientes con cualquier alergia diferente

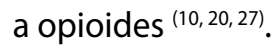

Finalmente, puede que el paciente desarrollara una pseudoalergia, definida como una reacción inmune no-anafiláctica ${ }^{(6)}$. La pseudoalergia y la anafilaxis son clínicamente indistinguibles, los signos y síntomas son prácticamente idénticos ${ }^{(14,25)}$ y causan eventos como eritema multiforme ${ }^{(15,22)}$ o neumonitis ${ }^{(29)}$. En ausencia de pruebas clínicas, Li et al., encontraron que el angioedema y la hipotensión fueron significativamente más altas en pacientes con verdadera alergia a opioides ${ }^{(11,30)}$.

\section{CONCLUSIONES}

Los pacientes oncologicos reciben múltiples medicamentos que pueden incluir opioides. Estos pueden causar efectos secundarios y, además, pueden causar reacciones anafilácticas difíciles de tratar. Cuando estos pacientes desarrollan una reacción alérgica severa es difícil establecer el agente causal de la anafilaxia durante la emergencia y las pruebas cutáneas no son siempre confiables. Esta secuencia de eventos lo cual puede conllevar a retrasos, complicaciones o interrupciones de los manejos establecidos en pacientes oncológicos, puede conllevar a retrasos, complicaciones o interrupciones de los manejos establecidos.

\section{REFERENCIAS BIBLIOGRÁFICAS}

1. Patanwala $A E$, Keim SM, Erstad BL. Intravenous opioids for severe acute pain in the emergency department. The Annals of pharmacotherapy. 2010; 44(11): 1800-1809. https://doi.org/10.1345/aph.1P438

2. Meng J., Rotiroti G, Burdett E, Lukawska JJ. Anaphylaxis during general anaesthesia: experience from a drug aller- gy centre in the UK. Acta anaesthesiologica Scandinavica. 2017; 61(3): 281-289. https://doi.org/10.1111/aas.12858

3. Ariosto D. Factors Contributing to CPOE Opiate Allergy Alert Overrides. AMIA Annu Symp Proc [Internet] 2014; 2014: 256-265. Disponible en: https://www.ncbi.nlm. nih.gov/pmc/articles/PMC4419937/

4. Kartal M, Oktay C, Bacanlı A, karadeniz Ö. A Rare Diagnosis in Emergency Department: Morphine Related Anaphylactoid Reaction. Turkish Journal of Emergency Medicine [Internet] 2009; 9(4): 181-183. Disponible en: https://www.turkjemergmed.com/abstract/331

5. Montañez MI, Mayorga C, Bogas G, Barrionuevo E, Fernandez-Santamaria R, Martin-Serrano A, et al. Epidemiology, Mechanisms, and Diagnosis of Drug-Induced Anaphylaxis. Front. Immunol. 2017 May 29; 8:614. doi: 10.3389/fimmu.2017.00614

6. Baldo BA, Pham NH. Histamine-releasing and allergenic properties of opioid analgesic drugs: resolving the two. Anaesth Intensive Care. 2012; 40(2): 216-235. https:// doi.org/10.1177/0310057X1204000204

7. Zhang B, Li Q, Shi C, Zhang X. Drug-Induced Pseudoallergy: A Review of the Causes and Mechanisms. Pharmacology. 2018; 101(1-2): 104-110. https://doi. org $/ 10.1159 / 000479878$

8. Gilbar PJ, Ridge AM. History of opioid allergy: what significance? J Oncol Pharm Pract. 2004; 10(3): 183-6. https:// doi.org/10.1191/1078155204jp131oa

9. Kemp SF, Lockey RF. Anaphylaxis: a review of causes and mechanisms. The Journal of allergy and clinical immunology. 2002; 110(3): 341-348. https://doi.org/10.1067/ mai.2002.126811

10. Mori F, Barni S, Manfredi M, Sarti L, Pecorari L, Pucci N, et al. Anaphylaxis to Intravenous Tramadol in a Child. Pharmacology. 2015; 96(5-6), 256-258. https://doi. org/10.1159/000441005

11. Hallberg P, Brenning G. Angioedema induced by tramadol--a potentially life-threatening condition. European journal of clinical pharmacology, 2005; 60(12): 901-903. https://doi.org/10.1007/s00228-004-0882-5

12. Li PH, Ue KL, Wagner A, Rutkowski R, Rutkowski K. Opioid Hypersensitivity: Predictors of Allergy and Role of Drug Provocation Testing. The journal of allergy and clinical immunology. In practice. 2017; 5(6): 1601-1606. https:// doi.org/10.1016/j.jaip.2017.03.035

13. Nasser SM, Ewan PW. Opiate-sensitivity: clinical characteristics and the role of skin prick testing. Clinical and experimental allergy: journal of the British Society for Allergy and Clinical Immunology. 2001; 31(7), 10141020. https://doi.org/10.1046/j.1365-2222.2001.01090.x

14. Baldo BA. Opioid Analgesic Drugs: Misuse, Toxicity, and Hypersensitivity. The journal of allergy and clinical immunology: In practice. 2017; 5(6): 1607-1608. https:// doi.org/10.1016/j.jaip.2017.04.028

15. Sánchez-González MJ, Barbarroja-Escudero J, Antolín-Amérigo $D$, Rodríguez-Rodríguez $M$, Pericet $L$, Medina l, et al. Erythema Multiforme Induced by Tramadol: An Allergy Assessment. Journal of investigational allergology \& clinical immunology. 2020; 30(4): 290-291. https://doi.org/10.18176/jiaci.0495

16. Sahutoglu C, Kocabas S, Askar FZ. Tramadol use in a patient with Brugada syndrome and morphine allergy: a case report. Journal of pain research. 2018; 11: 191-194. https://doi.org/10.2147/JPR.S150905 
17. Wahler RG, Smith DB, Mulcahy KB. Nebulized Fentanyl for Dyspnea in a Hospice Patient with True Allergy to Morphine and Hydromorphone. Journal of pain \& palliative care pharmacotherapy. 2017; 31(1): 38-42. https:// doi.org/10.1080/15360288.2017.1279499

18. Kalangara J, Potru S, Kuruvilla M. Clinical Manifestations and Diagnostic Evaluation of Opioid Allergy Labels - A Review. Journal of pain \& palliative care pharmacotherapy. 2019; 33(3-4): 131-140. https://doi.org/10.1080/153 60288.2019.1666955

19. Li PH, Ue KL, Wagner A, Rutkowski R, Rutkowski K. Opioid Hypersensitivity: Predictors of Allergy and Role of Drug Provocation Testing. The journal of allergy and clinical immunology: In practice. 2017; 5(6), 1601-1606. https:// doi.org/10.1016/j.jaip.2017.03.035

20. Sommerfield DL, Sommerfield A, Schilling A, Slevin $L$, Lucas M, Von Ungern-Sternberg BS. Allergy alerts - The incidence of parentally reported allergies in children presenting for general anesthesia. Paediatric anaesthesia. 2019; 29(2): 153-160. https://doi.org/10.1111/ pan. 13541

21. Kalangara J, Potru S, Kuruvilla M. Clinical Manifestations and Diagnostic Evaluation of Opioid Allergy Labels - A Review. Journal of pain \& palliative care pharmacotherapy. 2019; 33(3-4): 131-140. https://doi.org/10.1080/153 60288.2019.1666955

22. Powell MZ, Mueller SW, Reynolds PM. Assessment of Opioid Cross-reactivity and Provider Perceptions in Hospitalized Patients With Reported Opioid Allergies. The Annals of pharmacotherapy. 2019; 53(11): 1117-1123. https://doi.org/10.1177/1060028019860521
23. Benkhaial A, Kaltschmidt J, Weisshaar E, Diepgen TL, Haefeli WE. Prescribing errors in patients with documented drug allergies: comparison of ICD-10 coding and written patient notes. Pharmacy world \& science: PWS. 2009; 31(4): 464-472. https://doi.org/10.1007/s11096009-9300-5

24. Cabrera-Freitag P, Gastaminza G, Goikoetxea MJ, Lafuente A, De la Borbolla JM, Sanz ML. Immediate allergic reaction to atropine in ophthalmic solution confirmed by basophil activation test. Allergy. 2009; 64(9): 1388-1389. https://doi.org/10.1111/j.1398-9995.2009.02052.x

25. Brockow K, Romano A. Skin tests in the diagnosis of drug hypersensitivity reactions. Current pharmaceutical design. 2008; 14(27): 2778-2791. https://doi. org/10.2174/138161208786369821

26. Dewachter P, Lefebvre D, Kalaboka S, Bloch-Morot E. An anaphylactic reaction to transdermal delivered fentanyl. Acta anaesthesiologica Scandinavica. 2009; 53(8): 10921093. https://doi.org/10.1111/j.1399-6576.2009.02022.x

27. Baldo BA, Harle DG. Drug allergenic determinants. Monographs in allergy. 1990; 28: 11-51.

28. López-Marina V, Pizarro-Romero G, Costa-Bardají N. Neumonitis por hipersensibilidad asociada a tramadol [Hypersensitivity pneumonitis associated to tramadol]. Medicina clinica. 2006; 126(2): 77-78. https://doi. org/10.1157/13083574

29. Casale TB, Bowman S, Kaliner M. Induction of human cutaneous mast cell degranulation by opiates and endogenous opioid peptides: evidence for opiate and nonopiate receptor participation. The Journal of allergy and clinical immunology. 1984; 73(6), 775-781. https://doi. org/10.1016/0091-6749(84)90447-0 


\section{ANEXO 1}

Tabla de paraclínicos hematología

\begin{tabular}{lccccccc}
\hline Fecha & HB & Hct & Leucos & Linfos & Neutros & Eos & Plaq \\
\hline $30 / 04 / 2018$ & 12,1 & $36,7 \%$ & 4190 & 1930 & 480 & - & 494000 \\
$3 / 05 / 2018$ & 11,7 & $35,2 \%$ & 4660 & 2060 & 1280 & - & 417000 \\
$23 / 05 / 202$ & 8,8 & $28,1 \%$ & 2090 & 230 & 1520 & - & 64.000 \\
$13 / 06 / 2018$ & 12 & $36,4 \%$ & 6200 & 2250 & 3080 & 70 & 456.000 \\
$27 / 06 / 2018$ & 6.9 & $22 \%$ & 160 & 40 & 60 & 0 & 39.000 \\
$3 / 07 / 2018$ & 9,7 & $29,1 \%$ & 910 & 70 & 680 & & 41.000 \\
\hline
\end{tabular}

\section{ANEXO 2}

Algortimo de Naranjo y col., para medicamentos

\begin{tabular}{|c|c|c|c|c|}
\hline Metrotexate & Sí & NO & NS & Puntos \\
\hline ¿Existen notificaciones concluyentes sobre esta reacción? & 1 & & & \\
\hline ¿Se produjo la RA después de administrar el fármaco sospechoso? & & 0 & & \\
\hline ¿Mejoró la RA tras suspender la administración del fármaco o tras adminstrar un antagonista específico? & & 0 & & \\
\hline ¿Reapareció la RA tras readministración del fármaco? & & 0 & & \\
\hline ¿Existen causas alternativas (diferentes del fármaco) que podrían haber causado la reacción po si misma? & & & 0 & \\
\hline ¿Reapareció la RA tras administrar placebo? & & & 0 & \\
\hline ¿Se detectó el fármaco en la sangre (o en otros fluidos) en concentraciones tóxicas? & & & 0 & \\
\hline ¿Fue la reacción más severa al aumentar la dosis o menos severa al disminuirla? & & 0 & & \\
\hline $\begin{array}{l}\text { ¿Tuvo el paciente alguna reacción similar causada por el mismo fármaco u otro semejante en cualaquier } \\
\text { exposición anterior? }\end{array}$ & 1 & & & \\
\hline ¿Se confirmó el acontecimiento adverso por cualquier tipo de evidencia objetiva? & 1 & & & \\
\hline Puntuación total & 3 & & & \\
\hline
\end{tabular}

\begin{tabular}{|c|c|c|c|c|}
\hline Piperacilina tazobactam & Sí & NO & NS & Puntos \\
\hline ¿Existen notificaciones concluyentes sobre esta reacción? & 1 & & & \\
\hline ¿Se produjo la RA después de administrar el fármaco sospechoso? & & 0 & & \\
\hline ¿Mejoró la RA tras suspender la administración del fármaco o tras adminstrar un antagonista específico? & & 0 & & \\
\hline ¿Reapareció la RA tras readministración del fármaco? & & 0 & & \\
\hline ¿Existen causas alternativas (diferentes del fármaco) que podrían haber causado la reacción po si misma? & & & 0 & \\
\hline ¿Reapareció la RA tras administrar placebo? & & & 0 & \\
\hline ¿Se detectó el fármaco en la sangre (o en otros fluidos) en concentraciones tóxicas? & & & 0 & \\
\hline ¿Fue la reacción más severa al aumentar la dosis o menos severa al disminuirla? & & 0 & & \\
\hline $\begin{array}{l}\text { ¿Tuvo el paciente alguna reacción similar causada por el mismo fármaco u otro semejante en cualaquier } \\
\text { exposición anterior? }\end{array}$ & 1 & & & \\
\hline ¿Se confirmó el acontecimiento adverso por cualquier tipo de evidencia objetiva? & 1 & & & \\
\hline Puntuación total & 3 & & & \\
\hline
\end{tabular}




\begin{tabular}{|c|c|c|c|c|}
\hline Mercaptopurina & Sí & NO & NS & Puntos \\
\hline ¿Existen notificaciones concluyentes sobre esta reacción? & 1 & & & \\
\hline ¿Se produjo la RA después de administrar el fármaco sospechoso? & & 0 & & \\
\hline ¿Mejoró la RA tras suspender la administración del fármaco o tras adminstrar un antagonista específico? & & 0 & & \\
\hline ¿Reapareció la RA tras readministración del fármaco? & & 0 & & \\
\hline ¿Existen causas alternativas (diferentes del fármaco) que podrían haber causado la reacción po si misma? & & & 0 & \\
\hline ¿Reapareció la RA tras administrar placebo? & & & 0 & \\
\hline ¿Se detectó el fármaco en la sangre (o en otros fluidos) en concentraciones tóxicas? & & & 0 & \\
\hline ¿Fue la reacción más severa al aumentar la dosis o menos severa al disminuirla? & & 0 & & \\
\hline $\begin{array}{l}\text { ¿Tuvo el paciente alguna reacción similar causada por el mismo fármaco u otro semejante en cualaquier } \\
\text { exposición anterior? }\end{array}$ & 1 & & & \\
\hline ¿Se confirmó el acontecimiento adverso por cualquier tipo de evidencia objetiva? & 1 & & & \\
\hline Puntuación total & 3 & & & \\
\hline
\end{tabular}

\begin{tabular}{|c|c|c|c|c|}
\hline Tramadol & Sí & NO & NS & Puntos \\
\hline ¿Existen notificaciones concluyentes sobre esta reacción? & 1 & & & \\
\hline ¿Se produjo la RA después de administrar el fármaco sospechoso? & & 0 & & \\
\hline ¿Mejoró la RA tras suspender la administración del fármaco o tras adminstrar un antagonista específico? & & 0 & & \\
\hline ¿Reapareció la RA tras readministración del fármaco? & & 0 & & \\
\hline ¿Existen causas alternativas (diferentes del fármaco) que podrían haber causado la reacción po si misma? & & & 0 & \\
\hline ¿Reapareció la RA tras administrar placebo? & & & 0 & \\
\hline ¿Se detectó el fármaco en la sangre (o en otros fluidos) en concentraciones tóxicas? & & & 0 & \\
\hline ¿Fue la reacción más severa al aumentar la dosis o menos severa al disminuirla? & & 0 & & \\
\hline $\begin{array}{l}\text { ¿Tuvo el paciente alguna reacción similar causada por el mismo fármaco u otro semejante en cualaquier } \\
\text { exposición anterior? }\end{array}$ & 1 & & & \\
\hline ¿Se confirmó el acontecimiento adverso por cualquier tipo de evidencia objetiva? & 1 & & & \\
\hline Puntuación total & 6 & & & \\
\hline
\end{tabular}

\begin{tabular}{|c|c|c|c|c|}
\hline Vancomicia & Sí & NO & NS & Puntos \\
\hline ¿Existen notificaciones concluyentes sobre esta reacción? & 1 & & & \\
\hline ¿Se produjo la RA después de administrar el fármaco sospechoso? & 1 & & & \\
\hline ¿Mejoró la RA tras suspender la administración del fármaco o tras adminstrar un antagonista específico? & & 0 & & \\
\hline ¿Reapareció la RA tras readministración del fármaco? & & & 0 & \\
\hline ¿Existen causas alternativas (diferentes del fármaco) que podrían haber causado la reacción po si misma? & & & 0 & \\
\hline ¿Reapareció la RA tras administrar placebo? & & & 0 & \\
\hline ¿Se detectó el fármaco en la sangre (o en otros fluidos) en concentraciones tóxicas? & & & 0 & \\
\hline ¿Fue la reacción más severa al aumentar la dosis o menos severa al disminuirla? & & 0 & & \\
\hline $\begin{array}{l}\text { ¿Tuvo el paciente alguna reacción similar causada por el mismo fármaco u otro semejante en cualaquier } \\
\text { exposición anterior? }\end{array}$ & & 0 & & \\
\hline ¿Se confirmó el acontecimiento adverso por cualquier tipo de evidencia objetiva? & 1 & & & \\
\hline Puntuación total & 3 & & & \\
\hline
\end{tabular}

